\title{
Waste in the Medicare Program: a National Cross-Sectional Analysis of 2017 Low-Value Service Use and Spending
}

J Gen Intern Med 36(8):2478-82

DOI: $10.1007 / \mathrm{s} 11606-020-06061-0$

(C) Society of General Internal Medicine 2020

\section{INTRODUCTION}

Low-value health care services offer patients little to no clinical benefit, increase spending, and may cause patient harm. The Choosing Wisely® campaign and other efforts to reduce health care spending have focused attention on reducing waste since low-value services were last cumulatively assessed in Medicare in 2009. ${ }^{1}$ Other studies have addressed different populations or explored predictors of low-value service use and spending. ${ }^{2-6}$ In this analysis, we provide updated national estimates of low-value service use and spending in Medicare in 2017.

\section{METHODS}

We used Medicare claims and enrollment data for $100 \%$ of fee-for-service beneficiaries aged 65 and older continuously enrolled in parts A, B, and D for two years in 2017: $15,168,134$ beneficiaries, requiring at least one and up to three years of claims history preceding the 2017 measurement year. We assessed 35 claims-based low-value service measures reflecting Choosing Wisely ${ }^{\circledR}$ recommendations and other professional guidelines using the Milliman MedInsight ${ }^{\circledR}$ Health Waste Calculator to categorize services as "wasteful," "likely wasteful," or "not wasteful." We conservatively defined low-value services as "wasteful" services with sufficient claims history, excluding services billed in the inpatient claims file because we could not attribute spending to the low-value service itself. To provide a range, we calculated wasteful spending two ways: at the claim-line-level (allowed amount from only the claim-line or revenue center corresponding to a wasteful service, as applicable based upon the claim type) and at the claim-level (allowed amount from an entire claim with least one claim-line corresponding to a wasteful service).

Received April 21, 2020

Accepted July 14, 2020

Published online July 29, 2020

\section{RESULTS}

Among the included beneficiaries, 5,389,619 (35.5\%) had at least one low-value service, accounting for 10 million distinct services ( 0.66 per capita) (Table 1$)$. The three most frequent services were as follows: opioids for acute low back pain (2.8 million, 28.5\%), preoperative baseline laboratory studies (2.6 million, $25.6 \%$ ), and oral antibiotics for acute upper respiratory or external ear infections (1.4 million, 13.9\%)-comprising over twothirds of low-value services measured $(68 \%)$.

Cumulative low-value service spending varied from $\$ 723$ million (\$48 per capita) at the claim-line-level to $\$ 2.1$ billion (\$140 per capita) at the claim-level (Table 2). Spending per service varied from $\$ 6.32$ for bleeding time testing at the claim-line-level to $\$ 7344.39$ for renal artery revascularization at the claim-level (Table 2).

At the claim-level, the three services representing the most spending were the following: preoperative baseline laboratory studies ( $\$ 980$ million, $46.1 \%$ ), opioids for acute low back pain (\$188 million, 8.8\%), and unnecessary colorectal cancer screening (\$143 million, 6.7\%) - comprising nearly twothirds of wasteful spending $(62 \%)$.

At the claim-line-level, the three services representing the most spending were the following: opioids for acute low back pain ( $\$ 188$ million, $26.0 \%$ ), concurrent use of two or more antipsychotic medications (\$94 million, $13.0 \%$ ), and unnecessary colorectal cancer screening (\$79 million, $11.0 \%$ )-comprising half of wasteful spending $(50 \%)$.

\section{DISCUSSION}

As of 2017, low-value services remain common and costly in Medicare. Over one-third of beneficiaries received at least one low-value service, resulting in excess spending and in potential patient harm (e.g., perforation, bleeding, or infection from unnecessary colorectal cancer screening). Utilization and spending are concentrated among a small subset of measured services, suggesting targeted opportunities for waste reduction. While the measures differ somewhat, our utilization and spending findings fall in a similar range to prior estimates in the Medicare population. ${ }^{1}$ 
Table 1 Low-Value Service Utilization Among Medicare Fee-For-Service Beneficiaries in 2017

\begin{tabular}{|c|c|c|c|c|}
\hline Measure & $\begin{array}{l}\text { Distinct } \\
\text { patients }\end{array}$ & $\begin{array}{l}\text { Low-value } \\
\text { services total }\end{array}$ & $\begin{array}{l}\% \text { of all low- } \\
\text { value services }\end{array}$ & $\begin{array}{l}\text { Services per } \\
1000 \text { patients }\end{array}$ \\
\hline Opioids for acute low back pain & $1,034,398$ & $2,840,389$ & 28.45 & 187.26 \\
\hline $\begin{array}{l}\text { Preoperative baseline laboratory studies in patients without significant } \\
\text { systemic illness before elective low-risk surgery }\end{array}$ & $2,005,139$ & $2,558,495$ & 25.63 & 168.68 \\
\hline Oral antibiotics for acute upper respiratory or external ear infections & $1,131,131$ & $1,384,931$ & 13.87 & 91.31 \\
\hline $\begin{array}{l}\text { NSAIDs in patients with hypertension, heart failure or chronic kidney } \\
\text { disease }\end{array}$ & 698,609 & 914,874 & 9.16 & 60.32 \\
\hline Unnecessary colorectal cancer screening in adults $50-75$ years & 444,658 & 479,535 & 4.80 & 31.61 \\
\hline Concurrent use of two or more antipsychotic medications & 38,944 & 444,505 & 4.45 & 29.31 \\
\hline Screening for vitamin D deficiency & 304,184 & 326,161 & 3.27 & 21.50 \\
\hline $\begin{array}{l}\text { Cervical cancer screening in women not at high risk with adequate } \\
\text { prior screening }\end{array}$ & 264,285 & 272,123 & 2.73 & 17.94 \\
\hline $\begin{array}{l}\text { Annual EKGs or cardiac screening in asymptomatic patients without } \\
\text { risk factors }\end{array}$ & 225,372 & 256,102 & 2.57 & 16.88 \\
\hline $\begin{array}{l}\text { Preoperative EKG, chest x-ray and PFTs in patients without } \\
\text { significant systemic illness before low-risk surgery }\end{array}$ & 128,395 & 136,516 & 1.37 & 9.00 \\
\hline $\begin{array}{l}\text { Cardiac stress testing or advanced imaging for asymptomatic patients } \\
\text { without risk factors }\end{array}$ & 89,878 & 93,046 & 0.93 & 6.13 \\
\hline $\begin{array}{l}\text { Carotid duplex ultrasound for simple syncope with normal } \\
\text { neurological exam }\end{array}$ & 49,704 & 51,073 & 0.51 & 3.37 \\
\hline Imaging for uncomplicated headache without neurological symptoms & 29,920 & 30,793 & 0.31 & 2.03 \\
\hline Imaging for acute low back pain without red-flag signs & 30,129 & 30,199 & 0.30 & 1.99 \\
\hline Imaging for uncomplicated acute rhinosinusitis & 24,778 & 26,063 & 0.26 & 1.72 \\
\hline Coronary angiography in asymptomatic patients without risk factors & 19,302 & 20,859 & 0.21 & 1.38 \\
\hline CT scans for emergency department evaluation of dizziness & 18,407 & 18,577 & 0.19 & 1.22 \\
\hline Antidepressant monotherapy in bipolar disorder & 9074 & 14,157 & 0.14 & 0.93 \\
\hline Brain imaging for simple syncope with normal neurological exam & 13,570 & 13,791 & 0.14 & 0.91 \\
\hline $\begin{array}{l}\text { PICCs in stage III-V chronic kidney disease patients without prior } \\
\text { nephrology consult }\end{array}$ & 12,689 & 13,465 & 0.13 & 0.89 \\
\hline $\begin{array}{l}\text { Immunoglobulin } \mathrm{G} \text { or immunoglobulin } \mathrm{E} \text { tests in the evaluation of } \\
\text { allergy }\end{array}$ & 11,221 & 12,137 & 0.12 & 0.80 \\
\hline CT head/brain for sudden onset hearing loss & 10,413 & 10,572 & 0.11 & 0.70 \\
\hline Electroencephalography for headaches & 8767 & 9011 & 0.09 & 0.59 \\
\hline Vertebroplasty & 5355 & 6264 & 0.06 & 0.41 \\
\hline Renal artery revascularization & 3098 & 3596 & 0.04 & 0.24 \\
\hline $\begin{array}{l}\text { Antibiotics for adenoviral conjunctivitis without secondary infection } \\
\text { or other conditions }\end{array}$ & 3492 & 3572 & 0.04 & 0.24 \\
\hline $\begin{array}{l}\text { Coronary artery calcium scoring for patients with known coronary } \\
\text { artery disease }\end{array}$ & 2983 & 2994 & 0.03 & 0.20 \\
\hline $\begin{array}{l}\text { Preoperative echocardiography or stress test before low- or } \\
\text { intermediate-risk non-cardiac surgery }\end{array}$ & 1997 & 2079 & 0.02 & 0.14 \\
\hline PFTs before cardiac surgery without respiratory disease or symptoms & 1864 & 1922 & 0.02 & 0.13 \\
\hline Routine diagnostic testing for chronic urticaria & 1654 & 1654 & 0.02 & 0.11 \\
\hline Arthroscopic lavage and debridement for knee osteoarthritis & 1437 & 1442 & 0.01 & 0.10 \\
\hline Bleeding time testing & 1160 & 1310 & 0.01 & 0.09 \\
\hline MRI of peripheral joints to monitor rheumatoid arthritis & 903 & 936 & 0.01 & 0.06 \\
\hline Multiple palliative radiation treatments in bone metastases & 481 & 493 & $<0.01$ & 0.03 \\
\hline $\begin{array}{l}\text { DEXA screening for osteoporosis in women younger than } 65 \text { or men } \\
\text { younger than } 70\end{array}$ & 475 & 479 & $<0.01$ & 0.03 \\
\hline Total & $5,389,619$ & $9,984,115$ & 100 & 658.23 \\
\hline
\end{tabular}

Our study has limitations. Claims-based low-value service measures are inherently under-inclusive, capturing only the fraction of low-value services with professional consensus that are measurable via claims. They may be over-inclusive if claims cannot reflect the circumstances or history that make a given service highvalue. We use two methods to calculate spending to present a potential range; both have limitations. Claimline-level spending is specific but may miss related services on other claim-lines, underestimating wasteful spending. Claim-level spending is more sensitive but may include unrelated services billed on the same claim, overestimating wasteful spending. Both methods miss related services or downstream events billed on other claims.

Our findings suggest that targeted interventions to reduce low-value services - particularly the narrow subset responsible for the majority of spending - could substantially reduce 
Table 2 Low-Value Service Spending Among Medicare Fee-For-Service Beneficiaries in 2017

\begin{tabular}{|c|c|c|c|c|c|c|c|c|}
\hline \multirow[b]{2}{*}{ Measure } & \multicolumn{4}{|c|}{ Claim-level spending (broad definition) } & \multicolumn{4}{|c|}{ Claim-line-level spending (narrow definition) } \\
\hline & $\begin{array}{l}\text { Spending, } \\
\text { total } \\
\text { (\$1 mil) }\end{array}$ & $\begin{array}{l}\% \text { of all } \\
\text { low-value } \\
\text { spending }\end{array}$ & $\begin{array}{l}\text { Spending } \\
\text { per service }\end{array}$ & $\begin{array}{l}\text { Spending } \\
\text { per } 1000 \\
\text { patients }\end{array}$ & $\begin{array}{l}\text { Spending, } \\
\text { total (\$1 } \\
\text { mil) }\end{array}$ & $\begin{array}{l}\% \text { of all } \\
\text { low-value } \\
\text { spending }\end{array}$ & $\begin{array}{l}\text { Spending } \\
\text { per service }\end{array}$ & $\begin{array}{l}\text { Spending } \\
\text { per } 1000 \\
\text { patients }\end{array}$ \\
\hline $\begin{array}{l}\text { Preoperative baseline } \\
\text { laboratory studies in patients } \\
\text { without significant systemic } \\
\text { illness before elective low-risk } \\
\text { surgery }\end{array}$ & 979.72 & 46.09 & 382.93 & $64,590.37$ & 40.81 & 5.64 & 15.95 & 2690.71 \\
\hline $\begin{array}{l}\text { Opioids for acute low back } \\
\text { pain }\end{array}$ & 187.94 & 8.84 & 66.17 & $12,390.21$ & 187.94 & 25.98 & 66.17 & $12,390.21$ \\
\hline $\begin{array}{l}\text { Unnecessary colorectal cancer } \\
\text { screening in adults } 50-75 \\
\text { years }\end{array}$ & 142.54 & 6.71 & 297.25 & 9397.36 & 79.48 & 10.99 & 165.75 & 5240.20 \\
\hline $\begin{array}{l}\text { Concurrent use of two or } \\
\text { more antipsychotic } \\
\text { medications }\end{array}$ & 141.94 & 6.68 & 319.32 & 9357.68 & 94.22 & 13.02 & 211.96 & 6211.47 \\
\hline $\begin{array}{l}\text { Cardiac stress testing or } \\
\text { advanced imaging for } \\
\text { asymptomatic patients without } \\
\text { risk factors }\end{array}$ & 81.51 & 3.83 & 876.04 & 5373.87 & 64.29 & 8.89 & 690.98 & 4238.69 \\
\hline $\begin{array}{l}\text { PICCs in stage III-V chronic } \\
\text { kidney disease patients with- } \\
\text { out prior nephrology consult }\end{array}$ & 79.96 & 3.76 & 5938.60 & 5271.79 & 53.90 & 7.45 & 4003.17 & 3553.68 \\
\hline $\begin{array}{l}\text { Preoperative EKG, chest x-ray } \\
\text { and PFTs in patients without } \\
\text { significant systemic illness } \\
\text { before low-risk surgery }\end{array}$ & 78.44 & 3.69 & 574.59 & 5171.46 & 4.52 & 0.62 & 33.08 & 297.76 \\
\hline $\begin{array}{l}\text { Coronary angiography in } \\
\text { asymptomatic patients without } \\
\text { risk factors }\end{array}$ & 77.08 & 3.63 & 3695.09 & 5081.43 & 49.08 & 6.78 & 2352.84 & 3235.59 \\
\hline $\begin{array}{l}\text { Annual EKGs or cardiac } \\
\text { screening in asymptomatic } \\
\text { patients without risk factors }\end{array}$ & 68.51 & 3.22 & 267.52 & 4516.94 & 5.83 & 0.81 & 22.76 & 384.34 \\
\hline $\begin{array}{l}\text { Screening for vitamin D } \\
\text { deficiency }\end{array}$ & 44.04 & 2.07 & 135.02 & 2903.30 & 6.39 & 0.88 & 19.58 & 420.95 \\
\hline $\begin{array}{l}\text { Carotid duplex ultrasound for } \\
\text { simple syncope with normal } \\
\text { neurological exam }\end{array}$ & 33.78 & 1.59 & 661.34 & 2226.81 & 9.37 & 1.30 & 183.51 & 617.91 \\
\hline $\begin{array}{l}\text { Cervical cancer screening in } \\
\text { women not at high risk with } \\
\text { adequate prior screening }\end{array}$ & 31.79 & 1.50 & 116.83 & 2096.04 & 16.05 & 2.22 & 59.00 & 1058.46 \\
\hline $\begin{array}{l}\text { NSAIDs in patients with } \\
\text { hypertension, heart failure or } \\
\text { chronic kidney disease }\end{array}$ & 28.57 & 1.34 & 31.23 & 1883.72 & 28.57 & 3.95 & 31.23 & 1883.72 \\
\hline Renal artery revascularization & 26.41 & 1.24 & 7344.39 & 1741.18 & 21.61 & 2.99 & 6009.81 & 1424.78 \\
\hline $\begin{array}{l}\text { Brain imaging for simple } \\
\text { syncope with normal } \\
\text { neurological exam }\end{array}$ & 17.72 & 0.83 & 1284.94 & 1168.28 & 2.43 & 0.34 & 176.52 & 160.49 \\
\hline $\begin{array}{l}\text { Imaging for uncomplicated } \\
\text { headache without neurological } \\
\text { symptoms }\end{array}$ & 17.42 & 0.82 & 565.60 & 1148.23 & 7.71 & 1.07 & 250.53 & 508.61 \\
\hline $\begin{array}{l}\text { Oral antibiotics for acute } \\
\text { upper respiratory or external } \\
\text { ear infections }\end{array}$ & 16.98 & 0.80 & 12.26 & 1119.52 & 16.98 & 2.35 & 12.26 & 1119.52 \\
\hline $\begin{array}{l}\text { CT scans for emergency } \\
\text { department evaluation of } \\
\text { dizziness }\end{array}$ & 16.92 & 0.80 & 910.87 & 1115.57 & 3.23 & 0.45 & 173.73 & 212.78 \\
\hline Vertebroplasty & 15.13 & 0.71 & 2415.53 & 997.54 & 12.43 & 1.72 & 1984.15 & 819.40 \\
\hline $\begin{array}{l}\text { Imaging for uncomplicated } \\
\text { acute rhinosinusitis }\end{array}$ & 10.17 & 0.48 & 390.28 & 670.61 & 3.14 & 0.43 & 120.61 & 207.24 \\
\hline $\begin{array}{l}\text { Imaging for acute low back } \\
\text { pain without red-flag signs }\end{array}$ & 6.40 & 0.30 & 211.91 & 421.91 & 2.82 & 0.39 & 93.50 & 186.15 \\
\hline $\begin{array}{l}\text { Electroencephalography for } \\
\text { headaches }\end{array}$ & 6.35 & 0.30 & 704.43 & 418.49 & 4.30 & 0.59 & 477.12 & 283.45 \\
\hline $\begin{array}{l}\text { CT head/brain for sudden on- } \\
\text { set hearing loss }\end{array}$ & 3.37 & 0.16 & 318.55 & 222.02 & 1.64 & 0.23 & 154.74 & 107.85 \\
\hline $\begin{array}{l}\text { Immunoglobulin } G \text { or } \\
\text { immunoglobulin E tests in the } \\
\text { evaluation of allergy }\end{array}$ & 2.46 & 0.12 & 203.06 & 162.48 & 0.84 & 0.12 & 69.34 & 55.48 \\
\hline $\begin{array}{l}\text { Arthroscopic lavage and } \\
\text { debridement for knee } \\
\text { osteoarthritis }\end{array}$ & 1.88 & 0.09 & 1307.13 & 124.27 & 1.88 & 0.26 & 1307.13 & 124.27 \\
\hline Bleeding time testing & 1.80 & 0.08 & 1376.75 & 118.90 & 0.01 & $<0.01$ & 6.32 & 0.55 \\
\hline
\end{tabular}


Table 2. (continued)

\begin{tabular}{|c|c|c|c|c|c|c|c|c|}
\hline \multirow[b]{2}{*}{ Measure } & \multicolumn{4}{|c|}{ Claim-level spending (broad definition) } & \multicolumn{4}{|c|}{ Claim-line-level spending (narrow definition) } \\
\hline & $\begin{array}{l}\text { Spending, } \\
\text { total } \\
\text { (\$1 mil) }\end{array}$ & $\begin{array}{l}\% \text { of all } \\
\text { low-value } \\
\text { spending }\end{array}$ & $\begin{array}{l}\text { Spending } \\
\text { per service }\end{array}$ & $\begin{array}{l}\text { Spending } \\
\text { per } 1000 \\
\text { patients }\end{array}$ & $\begin{array}{l}\text { Spending, } \\
\text { total (\$1 } \\
\text { mil) }\end{array}$ & $\begin{array}{l}\% \text { of all } \\
\text { low-value } \\
\text { spending }\end{array}$ & $\begin{array}{l}\text { Spending } \\
\text { per service }\end{array}$ & $\begin{array}{l}\text { Spending } \\
\text { per } 1000 \\
\text { patients }\end{array}$ \\
\hline $\begin{array}{l}\text { PFTs before cardiac surgery } \\
\text { without respiratory disease or } \\
\text { symptoms }\end{array}$ & 1.38 & 0.06 & 716.49 & 90.79 & 0.03 & $<0.01$ & 14.13 & 1.79 \\
\hline $\begin{array}{l}\text { Multiple palliative radiation } \\
\text { treatments in bone metastases }\end{array}$ & 1.35 & 0.06 & 2730.37 & 88.74 & 0.91 & 0.13 & 1854.31 & 60.27 \\
\hline $\begin{array}{l}\text { Preoperative } \\
\text { echocardiography or stress } \\
\text { test before low- or } \\
\text { intermediate-risk non-cardiac } \\
\text { surgery }\end{array}$ & 1.30 & 0.06 & 625.76 & 85.77 & 0.96 & 0.13 & 464.13 & 63.61 \\
\hline $\begin{array}{l}\text { Coronary artery calcium } \\
\text { scoring for patients with } \\
\text { known coronary artery disease }\end{array}$ & 0.92 & 0.04 & 308.16 & 60.83 & 0.76 & 0.11 & 254.64 & 50.26 \\
\hline $\begin{array}{l}\text { Antidepressant monotherapy } \\
\text { in bipolar disorder }\end{array}$ & 0.78 & 0.04 & 55.14 & 51.46 & 0.78 & 0.11 & 55.14 & 51.46 \\
\hline $\begin{array}{l}\text { Routine diagnostic testing for } \\
\text { chronic urticaria }\end{array}$ & 0.71 & 0.03 & 429.80 & 46.87 & 0.13 & 0.02 & 76.77 & 8.37 \\
\hline $\begin{array}{l}\text { MRI of peripheral joints to } \\
\text { monitor rheumatoid arthritis }\end{array}$ & 0.34 & 0.02 & 361.89 & 22.33 & 0.29 & 0.04 & 306.57 & 18.92 \\
\hline $\begin{array}{l}\text { Antibiotics for adenoviral } \\
\text { conjunctivitis without } \\
\text { secondary infection or other } \\
\text { conditions }\end{array}$ & 0.08 & $<0.01$ & 23.56 & 5.55 & 0.08 & 0.01 & 23.56 & 5.55 \\
\hline $\begin{array}{l}\text { DEXA screening for } \\
\text { osteoporosis in women } \\
\text { younger than } 65 \text { or men } \\
\text { younger than } 70\end{array}$ & 0.05 & $<0.01$ & 96.47 & 3.05 & 0.03 & $<0.01$ & 55.11 & 1.74 \\
\hline Total & 2125.74 & 100 & 212.91 & $140,145.38$ & 723.46 & 100 & 72.46 & $47,696.24$ \\
\hline
\end{tabular}

wasteful Medicare spending. Interventions focused on this subset of low-value services may allow greater near-term progress in reducing waste in the health care system.

Acknowledgments: Dr. Reid had full access to all the data in the study and takes responsibility for the integrity of the data analysis. The authors acknowledge Scot Hickey and Mark Totten for their research programming . Milliman provided RAND free access to the Milliman MedInsight ${ }^{\circledR}$ Health Waste Calculator and technical assistance.

Rachel O. Reid, MD, MS ${ }^{1,2,3}$

John N. Mafi, MD, MPH ${ }^{4,5}$

Lesley H. Baseman, B.A. ${ }^{1}$

A. Mark Fendrick, MD ${ }^{6,7}$

Cheryl L. Damberg, $P h D^{5}$

${ }^{1}$ RAND Corporation,

Boston, MA, USA

${ }^{2}$ Division of General Medicine and Primary Care, Brigham and Women's Hospital,

Boston, MA, USA

${ }^{3}$ Harvard Medical School,

Boston, MA, USA

${ }^{4}$ David Geffen School of Medicine, University of California,

Los Angeles, CA, USA

${ }^{5}$ RAND Corporation,

Santa Monica, CA, USA
${ }^{6}$ Department of Internal Medicine, University of Michigan,

Ann Arbor, MI, USA

${ }^{7}$ Institute for Healthcare Policy and Innovation, University of Michigan,

Ann Arbor, MI, USA

Corresponding Author: Rachel O. Reid, MD, MS; RAND Corporation Boston, MA, USA (e-mail: rreid@rand.org).

Funding Information Rachel Reid, Lesley Baseman, and Cheryl Damberg's work was supported through the RAND Center of Excellence on Health System Performance, which is funded through a cooperative agreement (1U19HS024067-01) between the RAND Corporation and the Agency for Healthcare Research and Quality. John Mafi is supported by the UCLA Clinical and Translational Science Institute (CTSI) KL2 Award KL2TROO1882 (PI: Mitchell Wong), Arnold Ventures, and the National Institutes on Aging R01AG05981501 (PI: Catherine Sarkisian).

\section{Compliance with Ethical Standards:}

Conflict of Interest: Dr. Fendrick is a co-developer of the Milliman MedInsight Health Waste Calculator and receives royalties from its sale. The authors have no other conflicts of interest to disclose.

Disclaimer: The content and opinions expressed in this publication are solely the responsibility of the authors and do not reflect the official position of the Agency for Healthcare Research and Quality or the U.S. Department of Health and Human Services. 


\section{REFERENCES}

1. Schwartz AL, Landon BE, Elshaug AG, Chernew ME, McWilliams JM. Measuring low-value care in Medicare. JAMA Intern Med 2014; 174(7):1067-1076.

2. Reid RO, Rabideau B, Sood N. Low-value health care services in a commercially insured population. JAMA Intern Med 2016;176(10):15671571.

3. Reid RO, Rabideau B, Sood N. Impact of consumer-directed health plans on low-value healthcare. Am J Manag Care 2017;23(12):741748.

4. Mafi JN, Russell K, Bortz BA, Dachary M, Hazel Jr WA, Fendrick AM. Low-cost, high-volume health services contribute the most to unnecessary health spending. Health Aff 2017;36(10):1701-1704.
5. Schpero WL, Morden NE, Sequist TD, Rosenthal MB, Gottlieb DJ, Colla CH. For Selected Services, Blacks And Hispanics More Likely To Receive Low-Value Care Than Whites. Health Aff 2017;36(6):1065-1069.

6. Schwartz AL, Jena AB, Zaslavsky AM, McWilliams JM. Analysis of physician variation in provision of low-value services. JAMA Intern Med 2019; 179(1):16-25.

Publisher's Note: Springer Nature remains neutral with regard to jurisdictional claims in published maps and institutional affiliations. 